\title{
Burn Injury because Gasoline and Fire
}

\author{
Siniša Franjić \\ Siniša Franjić \\ Independent Researcher
}

\author{
*Corresponding author \\ Dr. Siniša Franjić, Independent Researcher
}

Submitted: 11 Jun 2020; Accepted: 20 Jun 2020; Published: 27 Jun 2020

\begin{abstract}
Child abuse really has no limit. The most horrific form of abuse happened in summer 2019. The media reported that in Dnipro, Ukraine, several hooligans were pouring with gasoline an eight-year-old boy and then set him on fire. Instead of helping him, the hooligans fled. Fortunately, they were quickly identified and brought to police. The unlucky boy has burns on $35 \%$ of his body. Burns are very serious injuries that carry with them certain health consequences.

The aim of this paper is to explain to the reader what the official authorities should do when a crime like this occurs. Unfortunately, the victim will have lasting consequences for their own health. Perpetrators should be adequately punished. This means that the competent state attorney's office should consider bringing charges for the most serious form of attempted murder, although in that case there are elements of some other criminal acts.
\end{abstract}

Key words: Boy, Hooligans, Fire, Burn

Introduction

Advances in therapy strategies, due to improved understanding of resuscitation, enhanced wound coverage, better support of hypermetabolic response to injury, more appropriate infection control, and improved treatment of inhalation injury, based on better understanding of the pathophysiologic responses to burn injury have further improved the clinical outcome of this unique patient population over the past years [1].

The depth and severity of the burn are also determined by the ability of the contact material to transfer heat, a factor referred to as the specific heat. This is especially important in scald and contact burns. The knowledge about the material type allows for a more accurate estimate of tissue damage. Burn depth is determined by the time of exposure, the temperature at which the burn occurred, and the caloric equivalent of the burn media.

Another determinant of the severity of burn is the location of the burn wound and the age of the burned patient. The thickness of the skin layers increases from the age of 5 up to the age of 50 . In elderly patients, the thickness starts to decrease at the age of 65 . The epidermis can vary by location from 0.03 up to $0.4 \mathrm{~mm}$. Clinically, the severity of burn injury can be categorized by the differences in the tissue damage and is determined by the depth of the burn.

(a)I degree: superficial burn of the epidermis

First-degree burns are painful, erythematous, and blanch to the touch with an intact epidermal barrier. Examples include sunburn or a minor scald from a kitchen accident. These burns do not re- sult in scarring, and treatment is aimed at comfort with the use of topical soothing salves with or without aloe and oral nonsteroidal anti-inflammatory agents.

(b)IIa degree: burn including epidermis and superficial dermis

(c)IIb degree: burn including epidermis and deep dermis

Second-degree burns are divided into two types: superficial and deep. All second-degree burns have some degree of dermal damage, by definition, and the division is based on the depth of injury into the dermis. Superficial dermal burns are erythematous, painful, blanch to touch, and often blister. Examples include scald injuries from overheated bathtub water and flash flame burns. These wounds spontaneously re-epithelialize from retained epidermal structures in the rete ridges, hair follicles, and sweat glands in 1-2 weeks. After healing, these burns may have some slight skin discoloration over the long term. Deep dermal burns into the reticular dermis appear more pale and mottled, do not blanch to touch, but remain painful to pinprick. These burns heal in $2-5$ weeks by reepithelialization from hair follicles and sweat gland keratinocytes, often with severe scarring as a result of the loss of dermis.

(d)III degree: burn including epidermis and dermis and subcuticular layer

Third-degree burns are full thickness through the epidermis and dermis and are characterized by a hard, leathery eschar that is painless and black, white, or cherry red. No epidermal or dermal appendages remain; thus, these wounds must heal by reepithelialization from the wound edges. Deep dermal and full-thickness burns require excision with skin grafting from the patient to heal 
the wounds in a timely fashion.

(e)IV degree: all dermal layers including fascia, muscles, and/or bones

Fourth-degree burns involve other organs beneath the skin, such as muscle, bone, and brain.

\section{Etiology}

The etiology of the burn can play an important role in determining the treatment and the extent and type of underlying physiologic derangements [2]. Additionally, the physiology of the skin itself plays a role in determining the severity of injury, the systemic manifestations, and how the burn progresses. Despite technologic advances in many areas of burn care, clinical assessment of the wound remains the standard technique for determining burn wound size and depth. Ideally, someone with experience caring for burn wounds will perform this assessment. Assessment of the wound, its depth, and its etiology are critical, as these determine not only the type of treatment needed (surgery versus topical wound care) for the wound but also its healing potential. Typically it is accepted that burns that are going to heal within 2-3 weeks do so without hypertrophic scarring or severe functional impairment. However, there have been many studies that promote early s urgical excision and split-thickness skin grafting as a method for improving survival and other outcomes from major burns. The challenge now is determining which wounds will heal within 2-3 weeks (and thus have minimal scarring) and which wounds require skin grafting. To do this, one must have the clinical acumen to predict which wounds will take longer than 2-3 weeks to heal.

\section{Flame}

Flame and flash burns are the most prevalent burns admitted to burn centers in the USA representing almost half of admissions [2]. Flash burns generally occur due to the ignition of flammable gases such as natural gas, butane, propane, or gasoline. The vapors of these gases are significantly denser than air (3-4 times heavier for gasoline, 2 times for butane, and 1.5 times for propane) and therefore can accumulate in enclosed spaces. When these gases are used inappropriately as a fire accelerant or in the production of butane hash oil, there can be an explosion. The explosion of the highly flammable vapors leads to intense heat for a very short period of time. Accordingly flash burns tend to affect exposed skin that is not protected by clothing (unless the clothing itself ignites shifting a flash burn to a flame burn). Because of the quick exposure times, flash burns are often partial thickness and frequently do not require skin grafting, but can have high percentage total body surface area (TBSA) affected. Many flash burns, however, end up needing grafting. Additionally, they can cause upper airway burns, edema, and inhalation injury.

\section{Child}

The basic tenet is that grafting should reduce the ultimate scarring of the burn wound [3]. Small full-thickness burns that are localized in areas without functional importance and with surrounding loose skin can contract without major problems. Burns on the lower abdomen or buttocks can contract to leave small and hidden scars. One must also consider that small skin grafts in the middle of a large surface area may look worse than allowing the wound to contract. A common problem is the child with a chest and abdominal scald with all areas, but one small area healing in 2 weeks. Should that remaining open area be grafted or allowed to heal with the potential for hypertrophic scarring? A small graft in the middle of the upper chest is quite unsightly, so allowing it to heal and dealing with a small hypertrophic scar may be the better option.

Like most complex surgical problems, burns are most cost-effectively managed in dedicated programs [4]. The American Burn Association burn center transfer criteria include recommendations that all children with full-thickness components over $10 \%$ of the body surface are transferred to a burn center. Recent data has clearly demonstrated that survival is improved for burned children if they are managed in higher volume pediatric burn programs. For individual children, burn care is a highly organized process going from resuscitation through reintegration that can be described in four distinct clinical phases. A publically available set of clinical practice guidelines for burn care has been disseminated by the American military and has specific recommendations for initial pediatric care.

The systemic response to burning is driven by a combination of neurohormonal changes, fluid losses across wounds, local colonization and systemic infection, hypoprotinemia, and wound colonization and infection. The clinical consequences of these changes can be particularly exaggerated in young children, with some children suffering larger scald burns developing systemic inflammation and organ failures in the absence of sepsis. During the first $72 \mathrm{~h}$, a relative hypodynamic state is common, with a decrease in cardiac output and metabolic rate. In successfully resuscitated children, a sustained hypermetabolic response follows, This is characterized by a near doubling of cardiac output and resting energy expenditure. The magnitude of this response peaks in patients with injuries of $60 \%$ or more of the body surface at as high as twice the normal basal metabolic rate. The hypermetabolic response includes enhanced gluconeogenesis, insulin resistance, and increased protein catabolism. This catabolic state is particularly detrimental in children and requires nutritional monitoring and support. Usual nutritional targets are $150 \%$ of normal caloric and protein needs. There is a rich history of metabolic research in direct interventions to eliminate adverse components of hypermetabolic physiology, particularly protein catabolism. Beta-adrenergic agonists and antagonists, non-steroidal anti-infl ammatory agents, recombinant growth hormone, insulin-like growth factor-1, and anabolic steroids have all been trialed in recent decades to modify this physiology in children. The appropriate role of these substances is a controversial area in the field.

\section{Pathology}

The tissues of the body, including the skin, can sustain burn injuries by several mechanisms [5]. The most common types of burns are related to thermal damage, which occurs when a tissue's cooling 
ability fails to compensate for externally applied heat. The extent of damage depends on the applied temperature, the ability of the tissue to conduct away excess heat, and the time for which the heat is applied. With these concepts in mind, it should be evident that thermal burns can occur very quickly when contact is made with very hot objects/substances. Conversely, objects/substances that are hot, but not extremely hot, can still cause thermal burns so long as contact with the object is long enough to overcome the tissue's ability to cool itself. Another important concept is that skin surfaces of different body regions, and skin from individuals of different ages, can vary greatly in their ability to withstand thermal insult.

First-degree burns are the most superficial and involve the epidermis [6]. They appear as a red discoloration of the skin. An example of this would be sunburn without blister formation. Second-degree burns have deeper penetration of injury involving the epidermis and dermis causing blister formation. An example of this may occur when one touches a hot pot on a stove and pulls the hand away quickly. Third-degree burns are full thickness, involving the epidermis, dermis, and subcutaneous layer, and appear as collapsed blisters with skin sloughing and red-to-brown discoloration. Fourth-degree burns have even deeper penetration of damage, often with charring and exposed underlying tissue including bone. The bones may be fractured from intense heat and the internal organs may have a firm, discolored, and shrunken appearance.

Thermal burns sustained by fire may be accompanied by smoke inhalation. In general, most fatalities from house fires are caused by smoke inhalation. Decedents dying directly in house fires usually have mostly postmortem burns. Fire fatalities that occur outside in an open space often do not have associated significant smoke inhalation because the smoke rises rapidly and is not inhaled. In the case of a flash fire, inhaled super-heated gases damage the upper airways, including the laryngeal mucosa, and causing death from reflexive closure of the airway at the level of the vocal cords with asphyxia and eventual fatal arrhythmia. The effects of smoke inhalation are often reflected by the amount of carbon monoxide present in the blood. This depends on the nature of the burning material.

Child abuse by burning constitutes from $6 \%$ to $20 \%$ of child abuse cases [7]. It is therefore essential that forensic investigators can recognize those lesions that have been caused by the application of heat and also that they can recognize those situations that may be confused with thermal injury. Burns occur when the skin and the subcutaneous tissues are damaged by thermal energy. For this to occur, a number of criteria must be satisfied, and the injury that results will depend on which of those criteria occur and for how long that injury is inflicted. These criteria include the temperature that is applied to the skin, whether the skin is wet or dry, whether the heat is wet or dry, the thickness of the keratin covering the area over which that heat is maintained, and the duration of that contact. These can be summarized in considering the coefficient of thermal conductivity. The resulting skin lesion and any systemic sequelae are dependent on the summation of these different components.
Dry burning results when the heat that is absorbed by the skin results in local tissue damage. If this damage is extensive enough, it may be associated with systemic responses which may be fatal if severe or untreated. Similar results may follow scalding, and given that liquids may, by their very nature, spread this contact effect over extensive areas of skin, scalding is a more common cause for hospital admission than injury inflicted by dry heat. The damage that occurs after thermal contact will initially be observed as reddening of the surface as cutaneous erythema occurs. If the temperature is high enough, the contact long enough, or the heat transfer deep enough, there will be damage to the dermal and subcutaneous tissues resulting in blistering and even ulceration. It is possible in extreme cases to have charring of the skin if the heat is so high that the organic material combusts, such as when fireworks explode or cigarette ends are applied to the skin for a prolonged time.

Thermal damage is traditionally classified according to whether the injury is inflicted by dry heat (burning) or by hot liquids (scalding), although the principles of damage are the same and it is not necessarily helpful to maintain this distinction. There are patterns of burn injury that are suggestive of an abusive origin. Many of these relate to the depth of the wound. Second- or third-degree burns, especially if they are multiple or symmetrical, should raise the index of suspicion. Skin is an effective insulator of heat, and most damage is confined to the epidermis due to the usually brief duration of contact. It is a normal reflex to rapidly withdraw an exposed area from applied heat, and if the damage is observed to be deep, then it implies that the time of contact may have been prolonged. This is strongly supportive of a non accidental cause. Burn injury patterns have been variously categorized, but one of the most useful classifications depends on the method of heat application rather than the resulting appearance.

\section{Management}

How well a burn heals depends on the severity of injury [8]. Clearly, superficial and small wounds do well no matter what we do to them. Very deep burns are much more difficult to manage and lead to more scarring. Severity is not only dependent upon depth, but also on the size of the burn injury. Clearly, a patient with $90 \%$ total body surface area (TBSA) full-thickness burns is going to have a greater challenge to heal his/her wounds. As a wound becomes massive, the "burden" of the injury tends to slow the healing process as the "supplies" needed for repair become more limited. The stress of the massive injury and the need to develop a "defense" against the invading organisms also make healing more challenging.

Burn depth has traditionally been divided into first-, second-, third-, and fourth-degree injuries. These categories are based on how deeply the injury penetrates the skin and subcutaneous tissue. In simple terms, skin is divided into the outer "epidermis," which acts as a barrier, and the "dermis", which provides the strength of skin. The skin covers the subcutaneous adipose tissue and the fat 
overlies the fascia, muscle, tendons, and bone. Depending on the depth of the burn, different strategies exist to close that injury with the least possible scarring. Physicians primarily categorize burns as having either partial thickness or full thickness [9]. Only an experienced medical practitioner can determine how deeply a burn has penetrated the skin, but there are some features of partial- and full-thickness burns that can be observed immediately after the incident:

- Patches of reddened skin that blanch with fingertip pressure and then refill are shallow partial-thickness burns. Blisters usually indicate deeper partial thickness burning, especially if the blisters increase in size just after the burn occurs.

- A leathery or dry surface with a color of white, tan, brown, red, or black indicates a full-thickness burn. The child feels no pain, because the nerve endings have been destroyed. Small blisters may be present but will not increase in size.

Investigators must develop good rapport with medical personnel, including both the hospital staff workers involved with the case and emergency medical technicians (EMTs). The EMTs, especially, can provide a wealth of information owing to the fact that they were probably the first persons to see the child's injuries. Also, an experienced social service investigator can provide valuable information regarding family hi s tory and any observed patterns of abuse.

Several factors affect the severity of a burn. A child's age plays a part in how severely the child is injured by a particular incident. For example, an adult will experience a significant injury of the skin after 1 minute of exposure to water at $127^{\circ} \mathrm{F}, 30$ seconds of e $\mathrm{x}$ posure at $130^{\circ} \mathrm{F}$, and 2 seconds of exposure at $150^{\circ} \mathrm{F}$. A child, however, will suffer a more severe burn in less time than an adult will, because children have thinner skin. As such, a young child's skin will be severely harmed even more rapidly and by less heat than will an older child's skin.

Nonaccidental trauma is an important consideration to help prevent future injury or death [10]. It is estimated that up to $20 \%$ of burn injuries are the result of child abuse or neglect, with highest incidence among young children (0-4 years of age). The likelihood of death is 4 times greater among those with suspected abuse. The anatomic location of the injury is affecting unreliable in differentiating nonaccidental and accidental burns; however, burns on both legs convey a 3 times greater likelihood of being abusive injury. Emergency physicians are mandatory reporters and do not need to definitively diagnose abuse but rather to reasonable cause to suspect abuse. Obtaining collateral information from social workers is beneficial because previous child protective services involvement is documented in $15 \%$ to $90 \%$ of reported nonaccidental burn cases. If there is any suspicion of nonaccidental trauma, the child should be admitted so that collateral information can be obtained while the child is safe.

The main goal of surgical treatment is the replacement of necrotic tissue [11]. One of the main problems encountered in extensively burned patients $>60 \%$ total body surface area (TBSA) is the scarcity of harvesting areas for autologous skin grafts.
Superficial dermal burns will heal without operation within 2-3 weeks, but deep dermal and full-thickness burn will require operation. It is widely accepted that if skin does not regenerate within 3 weeks, morbidity and scarring will be severe, so the trend in the treatment of deep dermal and full-thickness burns leans toward very early excision and grafting in order to reduce the risk of infection, decrease scar formation, shorten hospital stay and thereby reduce costs.

Excision of as much of the necrotic tissue as possible should be carried out whenever a patient is hemodynamically stable and the risks of the operation would not increase the mortality that would be expected from the traditional treatment. In patients with associated injuries such as inhalation injury, patients of extreme age, or patients with cardiac problems, special surgical treatment is required in deciding when and how much to excise.

\section{Reconstruction}

The skin is the largest organ, consisting of different layers [12]. Loss of skin integrity and skin functions due to injury or illness may acutely result in a substantial physiologic imbalance with long-term morbidity or even death. The most common cause of severe skin loss is thermal injury. Over the past decades, extraordinary advances have been made in the understanding of cellular and molecular processes of wound healing. This knowledge has led to wound care innovations and new developments in burn care, having even improved survival of severe burn injuries. The trend in current treatment regimens is beyond the preservation of life; the ultimate goal is to turn burn victims back into society, as full participants into their families and communities.

One of the milestones resulting in improved outcome was a more aggressive surgical approach to burn injuries; early wound debridement and early wound coverage has led to higher survival rates but also to a higher number of patients requiring postburn reconstruction. In the past, pediatric burn injuries were devastating and often fatal [13]. With improved burn resuscitation techniques in children, multidisciplinary approach to burn management and early excision and grafting, survival has become the norm. Now, with better survival, burn reconstruction has taken on a major role in the management of these patients. The basic concerns in pediatric burn reconstruction are function, comfort, and appearance. Normal and hypertrophic scarring, scar contractures, loss of anatomic structures, loss of function, and changes in color and texture of injured skin are common concerns among all burn patients and yet unique to each. To understand burn reconstruction, one must have a good understanding of wound healing and scar maturation in order to plan for adequate timing and reconstructive technique. The reconstructive ladder principle of starting simple when possible and progressing to more complex techniques is the basis for pediatric burn reconstruction. In all instances, the burn injury is assessed for the deficiency of tissue and distortion of anatomy. Traditionally, if there is no deficiency and local tissues are easily mobilized, excision and direct closure or Z-plasties can be performed. However, if there is deficiency in tissue, the need for skin replacement becomes critical. With advances in burn reconstruction, tis- 
sue expanders have become a great option not only for improved cosmetic results, but also for decrease in donor site morbidity. Tissue expanders are useful, particularly in the head and neck, especially in correction of burn-associated alopecia. This, however, involves multiple visits for expansion and a final surgery, weeks to months later for the reconstructive phase. Tissue expanders also have a high incidence of complications, including infection, tissue ischemia, and even extrusion of the expander, requiring removal and restarting the reconstructive process.

\section{Consequences}

A child's heart is less compliant than an adult's, and stroke volumes plateau at relatively low filling pressures [14]. This shifts the Starling curve to the left. Cardiac output (CO) depends almost exclusively on heart rate, and an immature heart is more sensitive to volume and pressure overload. Children are prone to the development of edema. They require particular monitoring for cerebral edema. The head needs to be elevated, especially in the first 24 to 48 hours after a burn injury. Frequent neurologic assessments are needed. Pulmonary edema is often caused by hydrostatic pressures, and in the absence of an inhalation injury, is almost diagnostic of fluid overload. Treatment of overhydration includes fluid restriction and diuresis.

Blood loss should be replaced on the second day after a burn injury. The usual amount of blood replacement in a 24-hour period is $10 \mathrm{ml} / \mathrm{kg}$, infused over 3 to 4 hours. Unless active blood loss is observed, no more than $15 \mathrm{ml} / \mathrm{kg}$ should be given over any 24-hour period. Larger quantities can result in cardiopulmonary congestion or severe hypertension. The mortality rate for all burn injuries is 4 $\%$. The average length of stay declined during this time period from 11 days, in the decade prior, to 9 days [15]. This is just over one day of hospitalization per $1 \%$ burn. As a result, these patients are being discharged with multiple, long term physical and psychological challenges, such as scarring, contractures, amputations, pain and poor psychological adjustment. In the past, most of the literature on burn injuries was devoted to the acute phase of hospitalization, particularly resuscitation efforts and surgical interventions. In recent years, issues associated with long term adjustment have been recognized as a priority for research and clinical practice. Because of the relative infancy of the field of burn rehabilitation, there is little research on the efficacy of interventions that can help with the long-term challenges, such as pain, depression and PTSD that these survivors face once they are discharged from the hospital.

The post-acute phase of recovery typically begins when patients leave the hospital and are challenged to reintegrate into society. For patients with severe burn injuries, this phase likely involves continued physical rehabilitation, along with possible continuation of procedures such as dressing changes and cosmetic surgery, all performed in outpatient clinics. Patients may encounter daily pain during rehabilitation, and they may also be forced to confront cosmetic or other existential changes that have occurred as a result of their injury. There is also increasing evidence that patients may develop chronic neuropathic pain. This is a period when patients slowly regain a sense of competence while sim ultaneously adjust- ing to the practical limitations of a burn injury. Studies have shown that the first year after hospitalization is a psychologically unique period of high distress.

\section{Trauma}

Trauma can be defined as bodily injury severe enough to pose a threat to life, limbs, and tissues and organs, which requires the immediate intervention of specialized teams to provide adequate outcomes [16]. Burn injury, unlike other traumas, can be quantified as to the exact percentage of body injured, and can be viewed as a paradigm of injury from which many lessons can be learned about critical illness involving multiple organ systems. Proper initial management is critical for the survival and good outcome of the victim of minor and major thermal trauma. However, even though burn injuries are frequent in our society, many surgeons feel uncomfortable in managing patients with major thermal trauma. Advances in trauma and burn management over the past three decades have resulted in improved survival and reduced mortality from major burns. Twenty-five years ago, the mortality rate of a $50 \%$ body surface area (BSA) burn in a young adult was about $50 \%$, despite treatment. Today, that same burn results in a lower than $10 \%$ mortality rate. Ten years ago, an $80-90 \%$ BSA burnyielded $10 \%$ survival. Today, over $50 \%$ ofthese patients aresurviving. Improved results are due to advancements in resuscitation, surgical techniques, infection control, and nutritional/metabolic support.

The skin is the largest organ in the body, making up 15\% of body weight, and covering approximately $1.7 \mathrm{~m} 2$ in the average adult. The function of the skin is complex: it warms, it senses, and it protects. A burn injury implies damage or destruction of skin and/or its contents by thermal, chemical, electrical, or radiation energies or combinations thereof. Thermal injuries are by far the most common and frequently present with concomitant inhalation injuries. Of its two layers, only the epidermis is capable of true regeneration. When the skin is seriously damaged, this external barrier is violated and the internal milieu is altered. The successful treatment of burn patientsincludes the intervention of a multidisciplinary burn team. The purpose of the burn center and the burn team is to care for and treat persons with dangerous and potentially disabling burns from the time of the initial injury through rehabilitation. The philosophy of care is based on the concept that each patient is an individual with special needs. Each patient's care, from the day of admission, is designed to return him or her to society as a functional, adaptable, and integrated citizen.

\section{Forensics}

Fatal and nonfatal exposure to heat and cold (thermal injury) is seen in the form of accidental injury as burns and scalds, as well as in the case of deliberate exposure with homicidal intent or for the purposes of concealing a homicide, e.g., homicidal arson vs. arson to conceal homicide [17]. Approximately 10-20\% of all abused children demonstrate heat-induced thermal injuries. Cold injuries, on the other hand, are rarely the result of a criminal act. In forensic medical practice, hypothermic fatalities raise the question of how the deceased came to be in a situation in which fatal hypothermia 
could occur. Most forms of thermal injury produce clearly differentiated findings. Fires involving naked flames tend to damage primarily unclothed areas of the body (head, hands, and legs). Flames traveling upwards will singe the hair, turning the ends yellowish gray and crinkly. Hot fluids, on the other hand, are absorbed by clothing, causing more severe injury to the skin in those areas where clothing is more fitted (upper body and belt area). Singed hair has never been seen in cases of scalding.

Unfortunately, scalding by hot liquids is a common childhood injury [18]. Not only the temperature of the fluid counts toward the severity, but the clothing worn, too. Scalding with close-clinging clothing cause more serious morphological changes, because the effect of the fluid is prolonged by being held in the cloth. The damage can be classified as previously into first, second and third degree burns, but fourth degree burns are never seen. The area affected is irregular, being scattered about the skin surface, and not uncommonly a child who has sat in hot water will show a "trousers-like" form to the damaged area of skin from below the waist.

Varying degrees of violence against children and adolescents is an age-old phenomenon in many countries and cultures [17]. The boundary between acceptable violence in the context of so-called necessary educational measures by parents or as part of accepted tradition, such as genital mutilation, and unacceptable violence leading to death or severe injury is not always clear. The association between chronic suffering in adults as a result of abuse suffered in childhood and adolescence is well known. A definition of child abuse could be formulated as follows:

Child abuse is non - random physical and / or mental damage inflicted on a child, either wilfully or through neglect, within the family or institutions, which causes injury and/or impaired development and which, in individual cases, may cause death .

Although the term "battered child" is often used to describe physical child abuse, the recent literature refers to non-accidental injury (NAI) and abusive or inflicted injury. The spectrum of violence against children covers blunt force (blows), thermal injury (burns, scalds), particular forms such as shaken baby syndrome, as well as the effects of psychological violence and neglect. The forensic clinical examination of children and adolescents (including the preservation of evidence) is subject to the same requirements as those for the examination of adults. Although police criminal statistics in numerous countries show several thousand cases of child abuse every year, the number of unknown cases is estimated to be far higher. It should be determined whether the suspect had a cause of action [19]. By occasion we mean the condition that immediately precedes the consequence, which creates the reciprocal effect of a larger range of conditions, and which immediately precedes the action, apparently manifesting itself as its actual cause. Furthermore, was the suspect's character highly inclined to commit such acts, or was the motive so strong that it broke the suspect's resistance? In fact, the question is, how did the motivation affect the suspect? Also important information is how much time has passed between the cause, the instigation of the act and the perprtration of the act.
The nature of the perpetrator is accessory to all other criteria in determining the intent to kill. In doing so, no abstract rules can be created because human nature is so created that it is often unpredictable. On the basis of the most banal reasons, conflicts arise in the overall constellation of interpersonal relationships that could least be expected.

\section{Conclusion}

Doctors and nurses in local hospital do everything what they can to help the boy recover as soon as possible. Unfortunately, certain consequences, above all, those of a psychological profile, will not disappear. The perpetrators of this crime are hooligans several years older than the victim. If they were able to pouring with gasoline an innocent boy and set him on fire, the question is what kind of misdoing are they still prepared to do. Criminal law can only treat this case as an attempted murder and cannot be treated differently. The perpetrators should be punished most severely for this crime.

\section{References}

1. Jeschke M G, Kamolz L P, Shahrokhi S (2013) Pathophysiology of Burn Injury: Burn Care and Treatment - A Practical Guide", Springer-Verlag, Wien, Austria 13-15.

2. Romanowski K, Greenhalgh D G (2016) Burn Pathophysiology: Burn Care for General Surgeons and General Practitioners, Springer International Publishing Switzerland, Cham, Switzerland 15-16.

3. Sheridan R L, Greenhalgh, D G (2016) Burns in Children: Burn Care for General Surgeons and General Practitioners, Springer International Publishing Switzerland, Cham, Switzerland 171-173.

4. Greenhalgh D G (2016) Operative Management of Burns“ in Greenhalgh, D. G. (ed): Burn Care for General Surgeons and General Practitioners", Springer International Publishing Switzerland, Cham, Switzerland 117.

5. Prahlow J (2010) Forensic Pathology For Police, Death Investigators, Attorneys And Forensic Scientists, Springer Science+Business Media, LLC, New York, USA 481.

6. Catanese C A, Catanese G (2010) Burns in Catanese, C. A. (ed): Color Atlas of Forensic Medicine and Pathology", CRC Press, Taylor \& Francis Group, Boca Raton, USA 353.

7. Ellis P (2014) Cutaneous Findings in Children" in Collins, K. A.; Byard, R. W. (eds): Forensic Pathology of Infancy and Childhood, Springer Science+Business Media New York, New York, USA 255-256.

8. Greenhalgh D G (2016) Wound Healing in Greenhalgh D. G. (ed): „Burn Care for General Surgeons and General Practitioners, Springer International Publishing Switzerland, Cham, Switzerland 95-97.

9. Swanson C R, Chamelin N C, Territo L, Taylor R W (2012) Criminal Investigation, Eleventh Edition“, McGraw-Hill, New York, USA 302.

10. Strobel A M, Fey R (2018) Emergency Care of Pediatric Burns" in Fox, S. M.; Woolridge, D. P. (eds): Pediatric Emergency 
Medicine“, Elsevier Inc., Philadelphia, USA 450.

11. Kamolz L P (2013) Acute Burn Surgery in Jeschke, M. G.; Kamolz, L. P.; Shahrokhi, S. (eds): Burn Care and Treatment - A Practical Guide“, Springer-Verlag, Wien, Austria 58.

12. Kamolz L P (2013) Burn Reconstruction“ in Kamolz, L. P.; Lumenta, D. B. (eds): Dermal Replacements in General, Burn, and Plastic Surgery - Tissue Engineering in Clinical Practice, Springer-Verlag Wien, Wien, Austria 211.

13. Glat P M, Azharian A, Hsu J F (2010) Pediatric Burn Reconstruction in Hyakusoku, H.; Orgill, D. P.; Téot, L.; Pribaz, J. J.; Ogawa, R. (eds): „Color Atlas of Burn Reconstructive Surgery, Springer-Verlag Berlin Heidelberg, Berlin, Germany 118.

14. Roth J J, Hughes W B (2016) The Essential Burn Unit Handbook, Second Edition", CRC Press, Taylor \& Francis Group, Boca Raton, USA 157-158.

15. Wiechman $S$ (2012) Long term consequences of burn injuries" in Kamolz, L. P.; Jeschke, M. G.; Horch, R. E.; Küntscher, M.; Brychta, P. (eds): „Handbook of Burns - Reconstruction and Rehabilitation, Volume 2", Springer-Verlag Wien, Wien, Austria 15.

16. Barret J P (2005) Initial Management and Resuscitation in Barret-Nerín J P, Herndon D N, rinciples and Practice of Burn Surgery", Marcel Dekker, New York, USA 1-2.

17. Dettmeyer R B, Verhoff M A, Schütz H F (2014) Forensic Medicine - Fundamentals and Perspectives", Springer-Verlag Berlin Heidelberg, Berlin, Germany 191-192 287. - 288.

18. Buris L (1993) Forensic Medicine, Springer-Verlag, Budapest, Hungary 143.

19. Pavišić B, Modly D, Veić P (2012) Kriminalistika - Knjiga 2 (Criminalistics - Book 2), Dušević\&Kršovnik, Rijeka, Croatia 48-49.
Copyright: (C2020 Siniša Franjić,, et al. This is an open-access article distributed under the terms of the Creative Commons Attribution License, which permits unrestricted use, distribution, and reproduction in any medium, provided the original author and source are credited. 